\title{
SOBRE EL ALCANCE DEL ANTIRREALISMO DE WITTGENSTEIN*
}

\section{On the Scope of Wittgenstein's Anti-realism}

\author{
Manuel Pérez Otero $a, b$ \\ http://orcid.org/0000-0002-0922-169X \\ perez.otero@ub.edu \\ ${ }^{a}$ Universidad de Barcelona, Barcelona, España \\ ${ }^{b}$ LOGOS (Research Group in Analytic Philosophy; http://www.ub.edu/grc_logos/ \\ manuel-perez-otero), Barcelona, España
}

\section{Resumen}

Karczmarczyck (2020) evalúa y critica algunas tesis centrales defendidas en mi libro Vericuetos de la filosofía de Wittgenstein en torno al lenguaje y el seguimiento de reglas (2018), centrado en las ideas del segundo Wittgenstein. Debato aquí sobre diversos puntos examinados por Karczmarczyck. En particular: (i) rechazo su concepción antirrealista de la intencionalidad, según la cual factores posteriores a una acción pueden determinar que dicha acción sea o no una acción deliberada; (ii) exploro otros aspectos de la contraposición realismo/antirrealismo, vinculándolos con la lectura antirrealista que Kripke, Karczmarczyck y otros autores hacen de Wittgenstein; (iii) explico por qué no es circular mi solución disposicional-teleológica al enigma sobre seguir una regla.

Palabras clave: Seguimiento de reglas; Kripke; Escepticismo semántico; Realismo; Deliberación racional; Condiciones de aseverabilidad; Intencionalidad.

\begin{abstract}
Karczmarczyck (2020) evaluates and criticizes some central theses defended in my book Vericuetos de la filosofía de Wittgenstein en torno al lenguaje y el seguimiento de reglas (2018), centered on the ideas of the second Wittgenstein. I discuss here various points examined by Karczmarczyck. In particular: (i) I reject his anti-realistic conception of intentionality, according to which factors occurring after a given action may determine whether or not it is a deliberate action; (ii) I explore other aspects

* Financiación: Proyectos "Etiología doxástica, conceptualizaciones singulares y la naturaleza de la filosofía” (PID2019-105728GB-I00), (MINECO/FEDER, UE) y "Perspectival Thoughts and Facts: New Questions" (FFI2016-81858-REDC; Red CONSOLIDER 2016, Acción de Dinamización, Red de Excelencia) (Gobierno de España/Unión Europea) / Grupo de investigación consolidado LOGOS (2017SGR63), AGAUR (Gobierno catalán).
\end{abstract}


of the realism/anti-realism contrast, linking them with the anti-realist reading that Kripke, Karczmarczyck and other authors make of Wittgenstein; (iii) I explain why my dispositional-teleological solution to the rule-following puzzle is not circular.

Key words: Rule-Following; Kripke; Meaning-Scepticism; Realism; Rational Deliberation; Assertability Conditions; Intentionality.

En un reciente artículo (Karczmarczyck, 2020), Pedro Diego Karczmarczyck evalúa y critica algunas tesis centrales defendidas en mi libro Vericuetos de la filosofía de Wittgenstein en torno al lenguaje y el seguimiento de reglas (Pérez Otero, 2018; en adelante Vericuetos). Agradezco a Karczmarczyck su interés por mi texto y a los editores de Análisis Filosófico que me den ahora la oportunidad de debatir con él sobre estos asuntos. Intentaré que la discusión contenga elementos atractivos adicionales a los que un lector pueda haber encontrado en esos dos escritos previos. Karczmarczyck presenta diversas observaciones sutiles y muy sugerentes. Me siento cercano a muchas de ellas, y lamentaría que algunos lectores pudieran llevarse una impresión desenfocada porque aquí las dejaré de lado, para explorar únicamente el territorio del desencuentro entre nuestras respectivas lecturas de Wittgenstein (y nuestras correspondientes posiciones filosóficas, ocasionalmente).

\section{Tiempo de deliberar, tiempo de hablar, tiempo de pensar}

Vericuetos se centra en la obra del segundo Wittgenstein, principalmente las Investigaciones filosóficas y Los cuadernos azul y marrón (Wittgenstein, 1953, 1958). Karczmarczyck desarrolla un análisis crítico de lo que considera mis “dos tesis principales del libro" (p. 93). La primera concerniría a cierta asimetría en los comportamientos intencionales de los sujetos. La segunda es una respuesta disposicionalteleológica al enigma sobre seguir una regla. Mis discrepancias con su lectura comienzan aquí. Hay en el libro cuatro tesis o propuestas originales sobre temas wittgensteinianos. Ciertamente, la última que se ofrece en Vericuetos es esa respuesta disposicional-teleológica. Pero la asimetría que resalto (relacionada con otra de mis propuestas principales) no es la que Karczmarczyck me atribuye. Veamos por qué.

En Vericuetos enfatizo la importancia de identificar una argumentación de Wittgenstein contra (lo que llamo) la tesis de la necesidad de la concepción mentalista-subjetivista sobre el significado, según la cual la comprensión del significado requiere asociar con las palabras entidades mentales subjetivas. Si la crítica wittgensteiniana 
está bien encaminada (y no son conceptualmente necesarias tales asociaciones), ¿en qué consistiría la comprensión del significado? Es claro que Wittgenstein vincula dicha comprensión con las acciones, o con las disposiciones o tendencias a actuar de ciertas maneras. Pero no podrían ser acciones cualesquiera. Se trataría de las acciones que alguien ajeno a Wittgenstein consideraría indicios de la comprensión: por ejemplo -y para casos suficientemente básicos- describir con sinceridad ciertas situaciones, y actuar conforme a lo que nos piden mediante oraciones imperativas. Surge de forma inmediata un potencial inconveniente. La comprensión semántica es compatible con mentir (entendamos por mentir decir lo contrario de lo que se cree), o con no hacer lo que se nos pide. Ante ese problema, ofrezco algunas reflexiones como posible línea de solución (no identifico en Wittgenstein ninguna respuesta concreta a ese problema; aunque Karczmarczyck, p. 105, afirma que le atribuyo esa solución). Al hilo de esas reflexiones, destaco ciertas asimetrías: hablar con sinceridad y mentir no tienen papeles simétricos en la explicación del conocimiento del significado; sucede lo mismo con obedecer una oración imperativa y no obedecerla. Que ciertas acciones (esencialmente vinculadas con la comprensión semántica) pudieran ser -en el marco wittgensteiniano- automáticas no implicaría que las acciones contrapuestas (mentir, desobedecer) también lo fueran. El contraste no es, contra la suposición de Karczmarczyck, entre acciones y omisiones. Es un contraste entre - por una parte- ciertas acciones, y -por otra- acciones diferentes u omisiones.

De todos modos, ese error no afecta al fondo de su crítica, que pone de manifiesto la contraposición entre concepciones más o menos realistas acerca de los estados intencionales. Yo señalo que mentir y desobedecer son respuestas típicamente mediadas por la deliberación racional (en tanto que comportamientos intencionales; obviamente, no hay deliberación si "desobedecemos" por estar dormidos cuando profieren ante nosotros una oración imperativa). ${ }^{1}$

${ }^{1} \mathrm{Si}$, según leo a Wittgenstein, en ciertos casos básicos de obedecer o hablar con sinceridad pudiera manifestarse una tendencia automática o "natural" del sujeto (aunque no sería una tendencia innata, sino resultante del aprendizaje de una lengua concreta), entonces mentir o (intencionalmente) desobedecer -tras deliberarcomportaría contrarrestar esa tendencia. No sé si eso implica, como sugiere Karczmarczyck (p. 105), que la deliberación se piense "como un mecanismo causal antinatural, como un proceso contra natura" en algún sentido tal vez reprobable del concepto.

Por otra parte, tampoco entiendo cuál es el intermediario que -según Karczmarczyck- yo postularía en relación con tales acciones; un intermediario también susceptible a las críticas de Wittgenstein al supuesto papel de las imágenes 
Karczmarczyck (y Wittgenstein según su interpretación; leyendo su texto, mi impresión es que suscribe todas las tesis que atribuye a Wittgenstein) propone una concepción antirrealista de estados y procesos mentales como deliberar. Según Karczmarczyck, la "autonomía de la gramática" defendida por Wittgenstein sugiere que preguntas como "¿en qué consiste la acción deliberada?" son irrelevantes o, al menos, comparativamente menos importantes que preguntas como "¿en qué circunstancias dirías que una acción es deliberada (en contraste con una que no lo es)?” (p. 106). Si se generaliza: propone anteponer “¿en qué circunstancias dirías que algo es F?" a “¿cuándo algo es F?".

Habría un enfoque meramente conceptual o lógico-gramatical (cercano -si no equiparable- a la perspectva que Wittgenstein llama simplemente "gramátical") que ordenaría así tales preguntas -concedo eso- según las perspectivas para su resolución de que dispone dicho enfoque. El debate realismo/antirrealismo es -entre otras cosas- sobre si también es sustantiva la pregunta “¿cuándo algo es F?" y si el enfoque lógico-gramatical basta siempre para resolverla, incluso pudiéndose descartar de antemano que se requiera para ello el auxilio de indagaciones empíricas (de la psicología, por ejemplo, si el tema es la deliberación o -en general- los estados mentales). Una concepción realista (o, al menos, compatible con el realismo) influida por Wittgenstein asignaría a la otra pregunta (la pregunta típicamente wittgensteiniana "¿en qué circunstancias dirías que algo es F?") un papel metodológico fundamental en la investigación; pero en la investigación de cuándo algo es $\mathrm{F}$.

Aplicar el enfoque "gramatical" conduciría-según Karczmarczycka la siguiente tesis: que una acción sea o no deliberada depende a veces de factores posteriores a la acción. Independientemente de lo que Wittgenstein pensara (luego vuelvo sobre eso), yo me siento cercano al realismo sobre los estados y procesos mentales que, contra las posiciones antirrealistas de notorios wittgensteinianos, mantenía -o eso parecía por esa época- Davidson (1963). En particular, que un acto sea o no deliberado no depende (ni metafísicamente, ni "gramaticalmente") de factores acaecidos con posterioridad a dicho acto. Irónicamente, creo que esta afirmación (sobre las relaciones entre la acción, la deliberación y el tiempo) es plenamente wittgensteiniana, resultante de consideraciones lógico-gramaticales autónomas, que -en este caso concreto- sí bastarían para establecer la cuestión. ${ }^{2}$

mentales (pp. 105-107). En algún momento de su texto se diría que invoco el intermediario para explicar la deliberación; en otros momentos parece referirse a la deliberación misma.

${ }^{2}$ Cualesquiera resultados empíricos supuestamente contrarios no concernirían 
Para apoyar su tesis sobre la posibilidad del carácter post facto de la diferencia entre actos deliberados y actos no deliberados, Karczmarczyck (pp. 95 y 106) trae a colación una reflexión del Cuaderno azul (remito a la paginación de la traducción al español mencionada en la bibliografía):

[...] el proceso que llamaríamos "hablar y pensar lo que se dice" no se distingue del de hablar sin pensar necesariamente por aquello que sucede mientras se habla. Lo que distingue a ambos puede muy bien ser lo que sucede antes o después de que se hable. (Wittgenstein, 1958, p. 73).

Detengámonos por un momento para reflexionar sobre ese caso. La cita no entra en conflicto con una visión realista tradicional de los estados y procesos mentales; es compatible, asimismo, con mi afirmación sobre las deliberaciones. Introduzcamos algo de la prosa bizantina que puede restar elegancia pero -conforme a la tradición analíticaaportarnos, a cambio, alguna luz. Wittgenstein se compromete con esta posición: Puede haber casos con las siguientes características: un sujeto, $S$, habla durante cierto período de tiempo, $t$; durante ese período, $t, S$ no está pensando (quizá deba entenderse: no hay, durante t, actividad de pensamiento relevantemente relacionada con la acción lingüística efectuada durante t; no estaba pensando "en lo que decía"); sin embargo, es incorrecto decir que $S$ habló sin pensar; que $S$ hablara durante $t y$ pensara lo que dijo puede depender -en parte-de algo sucedido antes o después de $t$.

En primer lugar, pensar no es lo mismo que deliberar. Conforme a los sentidos usuales de esos conceptos, deliberar implica pensar, pero no viceversa. Así, esa cita no permite concluir que Wittgenstein aplicara también específicamente a deliberar lo que afirma sobre pensar.

Por otra parte, si el factor determinante de hablar y pensar lo que se dice se sitúa antes de t, eso no comporta rechazar una visión realista tradicional de la intencionalidad. S puede prepararse muy bien las palabras que va a emitir en cierta ocasión, pensando en lo que dirá, incluso ensayando sus frases; cuando llega el momento, durante t, las

exactamente a las acciones y las deliberaciones; sino -tal vez- a algunos correlatos cercanos a ellas. Sucede algo similar cuando algunos psicólogos empíricos extraen consecuencias sustantivas sobre el libre albedrío basándose en (sus interpretaciones filosóficas de) experimentos solo relevantes para otros fenómenos meramente aledaños al mismo. Libet (1985) es un caso paradigmático de ese tipo de error metodológico, marcadamente antiwittgensteiniano. 
profiere sin estar pensando en lo que dice; sin embargo, sería incorrecto decir que "habló sin pensar lo que decía". Pensó lo que decía; pero lo pensó antes, no mientras lo decía.

No es tan fácil describir un caso en que el factor determinante sea posterior a t. Pero también parece factible. Consideremos dos situaciones alternativas, $\mathrm{C} 1$ y $\mathrm{C} 2$. S asiste a una reunión en la que intervienen varias personas, con tiempo limitado. Cuando le llega el turno, S habla durante t con precipitación, distraído y ofreciendo algún dato inexacto. En el subcaso C1, S no vuelve a hablar durante la reunión. En el subcaso C2, S, después de t (quizá ya ha intervenido otra persona), interrumpe la sesión, y vuelve a hablar para disculparse y corregir los datos incorrectos que había dado antes. Al describir la situación, dos días después, es natural decir respecto a $\mathrm{C} 1$, que $\mathrm{S}$ habló sin pensar lo que decía. Pero tal vez no sea correcto decir respecto a $\mathrm{C} 2$, que $\mathrm{S}$ habló sin pensar lo que decía, incluso bajo el supuesto de fondo: la acción lingüística a la que nos referimos (su hablar) es la que tuvo lugar durante t. Como ocurre cuando el factor determinante es previo a t, la clave para comprender estos casos es que gramaticalmente cabe aceptar la locución "hablar y pensar lo que se dice" sin presuponer que el tiempo del hablar deba coincidir con el tiempo del pensar lo dicho. Por eso el pensar sobre lo que se habla puede tener lugar antes o después del hablar. (Quizá tendríamos más reticencias ante la cita de Wittgenstein si hubiera empleado la frase "hablar pensando lo que se dice"; pero no usó esta última construcción, aunque sí la usa Karczmarczyck, p. 95). Por el contrario, creo que razones lógico-gramaticales establecen la imposibilidad de que el factor determinante del carácter deliberado de una acción sea posterior a la acción. ${ }^{3}$

\section{Diversidad del antirrealismo}

Será conveniente situar esas observaciones en una perspectiva más general sobre las controversias entre posturas realistas y posturas antirrealistas.

Los gritos y los quejidos no son verdaderos o falsos, pues ni siquiera son oraciones (por supuesto, pueden ser verdaderos conforme

\footnotetext{
${ }^{3}$ Estoy refiriéndome a las lecturas antirrealistas (de estados y procesos mentales, o de las ideas de Wittgenstein sobre esos ámbitos). En rigor, proponer que la diferencia entre una acción deliberada y una no deliberada puede depender del futuro es también compatible con una versión relativista del realismo (cf. nuestra próxima nota). Sucede algo similar respecto a ciertas interpretaciones antirrealistas de Wittgenstein (cf. la nota 6).
} 
a otro sentido del término; el sentido en que "verdadero" equivale a "auténtico" o a "genuino"). Tampoco tienen valor de verdad las oraciones imperativas, o las preguntas. Sería una cuestión menor clasificar a los enunciados como aquellas oraciones que meramente por sus rasgos gramaticales (en el sentido usual de "gramatical"; no tal como usa Wittgenstein el término) serían potencialmente susceptibles de poseer, en ocasiones particulares de uso, valores veritativos (o de tener condiciones de verdad), o bien reservar la etiqueta solo a las oraciones que -independientemente de esa apariencia gramatical- realmente tengan condiciones de verdad, y llamar pseudoenunciados a los restantes. Para fijar aquí la notación, optemos por la segunda opción.

Nuestros aparentes enunciados tratan de dominios muy diversos: astronomía, geología, química, matemáticas, seguimiento de reglas, significado, estados intencionales, valores (éticos, estéticos, y de otra índole), compras de bicicletas, etc. Así, nos topamos con la apariencia de que existen hechos relativos a esas temáticas; por ejemplo, hechos correlacionados con enunciados básicos verdaderos pertenecientes a esos campos. Sin embargo, diversas teorías filosóficas sostienen que algunos presuntos enunciados son pseudoenunciados, carecen de condiciones de verdad; no existirían, pues, hechos, relativos a los ámbitos supuestamente descritos por esos pseudoenunciados. En general, una teoría filosófica no veritativista sobre el tema $\mathrm{Q}$ afirma que los presuntos enunciados relativos al tema $\mathrm{Q}$ carecen de condiciones de verdad, y que no existen hechos sobre Q. Este último matiz, negar que haya hechos relativos a cierto ámbito, permite también hablar de antirrealismo sobre Q.

Las dificultades para identificar qué tipo de hechos objetivos determinan la verdad o falsedad de enunciados sobre el tema $Q$ puede alentar el no veritativismo sobre Q. Así, filósofos del Círculo de Viena (y no solo ellos) sostuvieron teorías no veritativistas sobre presuntos enunciados evaluativos de la ética y la estética; no habría hechos morales, ni hechos estéticos. ${ }^{4}$ Kripke, Karczmarczyck y otros comentaristas ven en Wittgenstein un antirrealismo generalizado sobre presuntos hechos concernientes a la semántica, la intencionalidad y el seguimiento de reglas. Los correspondientes presuntos enunciados serían pseudoenunciados, sin condiciones de verdad. Wittgenstein

\footnotetext{
${ }^{4}$ En Pérez Otero (2017, p. 120) explico por qué es menos apropiada la etiqueta con que se ha denominado tradicionalmente al no veritativismo: "no cognitivismo".

Ese texto aborda también una reacción diferente ante la dificultad que acabo de mencionar; ciertos filósofos optan por una teoría veritativista relativista sobre Q: hay verdad y falsedad, pero es relativa a factores adicionales, muy distintos de los que presupone una opción veritativista tradicional (o invariantista).
} 
rechazó el veritativismo respecto a varios tipos muy específicos de oraciones; por ejemplo, algunos casos de autoadscripciones de estados mentales. Pero el antirrealismo que le atribuyen estos autores es generalizado en el sentido de no restringirse a tales autoadscripciones; afectaría también a las atribuciones en segunda y tercera persona; es decir, tampoco existirían los hechos presuntamente expresados por las oraciones "Marta se estaba refiriendo a ti" o "Frege sabía sumar". Estas oraciones no serían verdaderas ni falsas. ${ }^{5}$ Por supuesto, las teorías no tradicionales (no invariantistas) sobre el significado de presuntos enunciados (sean teorías relativistas o antirrealistas) se comprometen con la viabilidad de elucidar cuál pueda ser dicho significado. Por ejemplo, pueden sostener que son -o son equivalentes a- otro tipo de oraciones (oraciones imperativas, pongamos por caso), o incluso que deben entenderse como gritos, quejidos, etc. Según los intérpretes de Wittgenstein recién mencionados, el filósofo austríaco propondría asignarles condiciones de aseverabilidad (en lugar de condiciones de verdad): condiciones bajo las cuales sería (o estimaríamos) apropiado proferir las correspondientes oraciones. (En realidad, sería mejor hablar de condiciones de felicidad de la proferencia, o algo semejante. Kripke (1982, p. 86; remito a la paginación de la traducción al español) reconoce que el término "aseverabilidad" aquí resulta desafortunado, precisamente por el punto principal que está en juego). ${ }^{6}$

Crispin Wright (1984, pp. 768-769) argumentó que el escepticismo antirrealista que Kripke atribuye a Wittgenstein se generalizaría a todos los enunciados, no solo los enunciados semánticos, intencionales o sobre seguir reglas; no habría hechos de ningún tipo (si acierta el Wittgenstein kripkeano), tampoco geológicos, químicos, etc. Su argumentación puede bloquearse, a mi juicio; pero el asunto dista de ser trivial. Un elemento insatisfactorio de Kripke (1982) es que no hace explícito si compartiría ese juicio de Wright. Puesto que Kripke circunscribe su análisis a

${ }^{5}$ Contemplo ahora como una insuficiencia de Vericuetos no haber separado-cuando se trataba de estudiar a Wittgenstein- ambos tipos de atribuciones (sobre cuestiones intencionales, semánticas y de seguimiento de reglas): las autoadscripciones y el resto. En cualquier caso, quiero ahora llamar la atención hacia otro contraste.

${ }^{6}$ Una teoría relativista sobre Q es -en principio- menos revisionista que una teoría no veritativista, pues no necesita postular que los presuntos enunciados acerca de $Q$ son pseudoenunciados; en otras palabras, preserva mejor -ceteris paribus-nuestros juicios intuitivos preteóricos. La preponderancia de Wittgenstein por el sentido común habla en contra de la interpretación antirrealista kripkeana; los factores que según el Wittgenstein kripkeano constituirían las condiciones de aseverabilidad de las oraciones problemáticas permitirían una interpretación veritativista, conforme a condiciones de verdad de tipo relativista. 
ciertas clases de enunciados (focalizando la discusión, particularmente, en oraciones sobre querer decir tal o cual cosa, o sobre seguir reglas), sería razonable contestar negativamente: seguramente Kripke no cree (o al menos no se habría planteado) que Wittgenstein propusiera ese antirrealismo global. Por razones emparentadas con este mismo punto, veo problemático atribuir a Wittgenstein el antirrealismo global. (Si esa posición se manifiesta -en especial- cuando el filósofo vienés aborda oraciones semánticas, intencionales o sobre seguir reglas, ¿por qué no se manifiesta también ejemplificándola con oraciones sobre el color de la nieve o la altura de un árbol?). Pero nos interesa también qué diría Karczmarczyck.

Aunque no estoy seguro de haber entendido sus reflexiones, cuando Karczmarczyck comenta mi discusión sobre conceptos cúmulo (Karczmarczyck, 2020, pp. 97-102), parecería que extiende el antirrealismo de Wittgenstein también -al menos- a todas aquellas oraciones que contengan términos que expresen ese tipo de conceptos (conceptos cúmulo). ¿Hay conceptos cúmulo fuera del campo de la semántica, las intenciones y el seguimiento de reglas? Si es así (y si he interpretado bien a Karczmarczyck), el dominio del supuesto antirrealismo wittgensteiniano se amplíaría mucho. Y podríamos preguntarnos: si afecta a cualquier término $\mathrm{F}$ que exprese un concepto cúmulo (de manera que no hay verdad ni falsedad al usar F), ¿por qué no debe generalizarse hasta obtener el antirrealismo global que Wright contempla como consecuencia de la lectura kripkeana de Wittgenstein?

Tiendo a pensar que el Wittgenstein real difiere del escéptico antirrealista generalizado sobre semántica, intencionalidad y seguimiento de reglas que postulan Kripke y Karczmarczyck. Es mayor mi convicción de que Wittgenstein no respaldaría el antirrealismo global; y (al margen de Wittgenstein) mucho mayor todavía mi rechazo a ese antirrealismo global, seguramente inconsistente (al menos desde un punto de vista pragmático). Me habría gustado ver con claridad por dónde trazan Kripke y Karczmarczyck la línea de demarcación del antirrealismo de Wittgenstein.

\section{Seguir una regla y la supuesta circularidad}

En el último capítulo de Vericuetos defiendo una solución disposicional-teleológica al enigma wittgensteiniano sobre seguir una regla examinado por Kripke (1982); una solución inmune a las críticas que dirige Kripke contra cierta solución disposicional simple. Según Karczmarczyck, mi solución encierra una circularidad viciosa y yo 
mismo así lo reconocería (cf. pp. 113-114 y 119). Discrepo de ambas tesis. Pero admito que a este respecto la exposición de mi propuesta tal vez haya sido poco clara (tanto en Vericuetos como en Pérez Otero, 2016, donde abordo con mayor detalle la solución disposicional-teleológica). Intentaré ahora contribuir a disipar la niebla. Mencionaré también otros puntos menores en que la lectura que hace Karczmarczyck de mi texto no me ha parecido acertada.

El propio Kripke presenta la paradoja wittgensteiniana (el enigma sobre seguir una regla) como si nos dirigiéramos inicialmente a un escéptico menos radicalizado que el escéptico cuyo reto se intenta analizar. El reto escéptico de fondo concierne a cualesquiera usos de nuestras palabras: pasados, presentes y futuros. Pero es aconsejable formular el desafío, al inicio, como si solo afectara a los usos pasados:

Para que el escéptico pueda siquiera conversar conmigo, hemos de tener un lenguaje común. Por tanto, estoy suponiendo que el escéptico, provisionalmente, no está poniendo en duda mi uso presente de la palabra "más" [...] Él se limita a poner en duda que mi uso presente concuerde con mi uso pasado, que yo esté en el momento presente actuando conforme a mis intenciones lingüísticas previas. (Kripke, 1982, p. 26)

Por supuesto, en último término, si el escéptico está en lo cierto, carecerían de sentido los conceptos de querer decir una de las funciones en lugar de la otra [...] si esto es correcto [...] no puede haber hecho alguno con respecto a cuál es la función que quise decir; y si no puede haber hecho alguno con respecto a cuál es la función que quise decir en el pasado, tampoco puede haberlo en el presente. Ahora bien, antes de segar la hierba bajo nuestros propios pies, empezamos hablando como si la noción de que en el momento presente queremos decir una cierta función mediante "más" no estuviera cuestionada y fuese incuestionable. Solo cuestionaremos los usos pasados. En otro caso, seremos incapaces de formular nuestro problema. (Kripke, 1982, pp. 27-28).

Tal vez Karczmarczyck considera circular mi solución porque me quedé en ese primer nivel de discusión. Era una solución apta para afrontar el reto escéptico comparativamente menos radical, que no cuestiona nuestro uso presente de las palabras. Aprovecho aquí la ocasión para exponer el asunto y explicitar cómo debería aplicarse y/o exponerse mi solución ante el escéptico genuino de la paradoja, 
que también pretende poner en duda los usos presentes del lenguaje. Llamemos a uno y otro, respectivamente, el escéptico inicial y el escéptico radical.

Mi propuesta, (DT), contiene estas cláusulas:

$\mathrm{Si}$ (i) en condiciones normales, $\mathrm{S}$ tiene la disposición teleológica a responder ante la pregunta por la suma " $x+y$ " en los casos básicos indicando cuál es, efectivamente, la suma de $x$ e $y$, (ii) [...], y (iii) la suma es la operación matemática más simple que extiende a los restantes casos (para el resto de pares de números naturales) esa disposición de $\mathrm{S}$, entonces $\mathrm{S}$ quiere decir la suma al usar signos como "más", "suma" y “+". (Pérez Otero, 2018, p. 101).

$\mathrm{El}$ antecedente de ese enunciado condicional invoca los conceptos de condiciones normales, casos básicos y suma. Por eso -según entiendodenuncia Karczmarczyck mi propuesta como circular (aunque solo objeta explícitamente al uso de los dos primeros conceptos). El escéptico inicial no pretende objetar a la legitimidad de invocar el concepto de suma. Y tampoco debería rechazar el uso de los otros dos conceptos, una vez clarificadas las posibles dudas con indicaciones relativamente poco controvertidas (algunas se ofrecen en Vericuetos). Por ejemplo, admito que (como recoge Karczmarczyck, p. 114) muy probablemente el concepto mismo de caso básico solo pueda explicarse mediante ilustraciones; y que será gradual la diferencia entre casos básicos y el resto de casos; una consecuencia -que ningún wittgensteiniano considerará problemáticaes la imprecisión de (DW).

No me queda claro si Karczmarczyck me achaca circularidad porque está pensando en el escéptico radical (enseguida hablo de él), o porque una frase suya (que contiene una cita de mi libro) no es una errata -como podría parecer- sino un error. Escribe Karczmarczyck:

La idea [la idea de mi propuesta] es que, a partir de ciertos casos básicos, "la inclinación natural a continuar de cierta manera coincidirá con la aplicación correcta de la regla" (Pérez Otero, 2018, p. 98). (Karczmarczyck, 2020, p. 114)

Pero no es esa la idea. Mi texto dice otra cosa:

Aunque cabe trazar una distinción entre aplicaciones correctas y aplicaciones incorrectas de la regla, eso solo es factible en la medida en que nos alejamos de los casos básicos o paradigmáticos. Para 
estos últimos, en cierto modo, la inclinación natural a reaccionar de cierta manera coincidirá con la corrección en la aplicación de la regla. (Pérez Otero, 2018, p. 98).

Con "estos últimos" me refiero en ese fragmento a los casos básicos o paradigmáticos; no a los casos alejados de los casos básicos o paradigmáticos, como parece interpretar Karczmarczyck. Y para los casos restantes (conforme nos alejamos de los casos básicos o paradigmáticos) es la simplicidad el factor crucial, determinante de qué regla se está siguiendo. ${ }^{7}$

¿Qué pasa con el escéptico radical? De nuevo, es relevante la dialéctica descrita certeramente por Kripke. Nos resulta inteligible el planteamiento del escéptico inicial. Un modo en que cierto sujeto $\mathrm{S}$ (por ejemplo, un escéptico inicial, o alguien que atiende a su reto) podría convertirse en escéptico radical sería tras concluir que, en efecto, ningún hecho determinaba qué queríamos decir con las palabras en nuestros usos pasados; entonces, tampoco habrá hecho alguno como base de nuestros usos presentes. Pero con mi propuesta -inteligible, sin circularidad, incluso para el escéptico inicial- pretendo legítimamente haber interceptado esa posible trayectoria de su pensamiento. (Por supuesto, en filosofía -y no solo en filosofía- tener esa pretensión de legitimidad al sostener un argumento que nos parece convincente no comporta la ingenuidad de creer que cualquier interlocutor va a aceptarlo y concordar con nosotros.)

Pero, ¿no podría $\mathrm{S}$ ser, ya desde el comienzo, un escéptico radical? Si fuera posible la existencia de un $\mathrm{S}$ con tales características, mi propuesta (DW) sería dialécticamente ineficaz para convencer a $\mathrm{S}$ de su error. Pero resultaría harto complicado inferir de ello (de ese enunciado condicional subjuntivo) alguna insuficiencia en (DW). Como sugiere Kripke, ese presunto $\mathrm{S}$ seguramente no sería capaz de formular ante nosotros su reto. Todo esto se vincula con dos puntos sobre los cuales no podremos extendernos aquí: las perspectivas de refutar mediante

${ }^{7}$ Remito a Pérez Otero (2018, pp. 109-112) al lector que ahora mismo tenga presente las objeciones de Kripke (1982) al uso del concepto de simplicidad para solucionar la paradoja. Por cierto, contrariamente a la lectura de Karczmarczyck, no "[me] esfuerz[o] por caracterizar una noción causal de la simplicidad" (p. 114; cf. también su nota 20). Ni me esforzaría, pues no serviría para mis propósitos. Las relaciones de mayor o menor simplicidad en que quiero basarme involucran entidades materiales complejas (disposiciones teleológicas) pero también entidades abstractas no materiales (en ese sentido entiendo las operaciones matemáticas que menciono); estas últimas no pueden intervenir -creo yo- en relaciones causales. 
algún tipo de argumentación trascendental la pretensión de ponerse en la posición de dicho $\mathrm{S}$; el carácter relativo de la eficacia dialéctica de los argumentos antiescépticos (he abordado ambos puntos en otros trabajos, que nos alejarían de las cuestiones wittgensteinianas objeto de nuestro debate actual).

Quizá pudiéramos concebir a ese sujeto $\mathrm{S}$ si asumimos otra simplificación: que $\mathrm{S}$ solo ponga en duda nuestros usos (pasados, presentes, futuros) de signos directamente relacionados con la suma; respecto al resto del vocabulario, tenemos ahora un lenguaje común (como indicaba Kripke). Tal vez sea posible esa posición escéptica. Entonces, a mi juicio, los detalles de cómo debería reformularse la idea principal contenida en (DW) para afrontar dicho reto dependerán de cómo consiguiera $\mathrm{S}$ presentar el enigma sobre la suma. (La reformulación de (DW), por ejemplo, no involucraría el concepto de suma en su antecedente; cobraría mayor relieve la invocación paradigmática de casos básicos; etc.).

Concluyo aludiendo a otro punto de discrepancia con Karczmarczyck. Sostiene que hago una lectura epistemológica del enigma wittgensteiniano (Karczmarczyck, 2020, pp. 115-118). No lo veo así. Mi lectura es plenamente ontológica o constitutiva (por usar sus propias palabras; p. 118), no epistemológica. Kripke enumera diversas objeciones a una solución disposicionalista simple, y en Vericuetos explico por qué una de ellas depende de presuponer un internismo epistemológico que no cabe atribuir a Wittgenstein. Pero eso no implica que "el punto fundamental sobre el que descansa [mi] respuesta al reto escéptico [sea] el rechazo del internismo epistemológico" (p. 115). Dicho rechazo es solo un elemento adicional de mi comprensión general de la problemática. Como menciona Karczmarczyck, Baker y Hacker (1984) lanzaron una acusación similar contra Kripke (indicando que el escepticismo semántico que había reconstruido era de tipo epistemológico); acusación errónea en ese caso, afirma Karczmarczyck. Salvando las distancias, me parece análogamente errónea su crítica contra mi interpretación del escepticismo semántico. Me cuesta ver qué elementos del libro de Kripke (/ de Vericuetos) inducen a creer a Baker y Hacker (/ a Karczmarczyck) que el texto aborda el enigma sobre seguir una regla solo desde una perspectiva epistemológica, no ontológica.

\section{Bibliografía}

Baker, G. P. \& Hacker, P. M. S. (1984). Scepticism, rules and language. Basil Blackwell. 
Davidson, D. (1963). Actions, reasons and causes. Journal of Philosophy, 60, pp. 685-700. Reimpreso en D. Davidson (1980). Essays on actions and events, Clarendon Press, pp. 3-19.

Karczmarczyck, P. D. (2020). Wittgenstein sin vericuetos: Escepticismo semántico y la autonomía de la gramática. Análisis Filosófico, 40(1), 93-121. https://doi.org/10.36446/af.2020.332

Kripke, S. (1982). Wittgenstein on rules and private languages: An elementary exposition. Harvard University Press. (Traducción: Wittgenstein a propósito de reglas y lenguaje privado: Una exposición elemental. Tecnos, 2006).

Libet, B. (1985). Unconscious cerebral initiative and the role of conscious will in voluntary action. Behavioral and Brain Sciences, 8(4), 529539 .

Pérez Otero, M. (2016). Respuestas disposicionalistas al problema wittgensteiniano-kripkeano sobre el significado y el seguimiento de reglas. Crítica. Revista Hispanoamericana de Filosofía, 48(143), 21-45. http://critica.filosoficas.unam.mx/index.php/critica/article/ view/216 ; 10.22201/iifs.18704905e.2016.216

Pérez Otero, M. (2017). La justificación de algunas teorías relativistas contemporáneas. Teorema, 36(2), 105-122.https://dialnet.unirioja. es/servlet/articulo?codigo $=6015371$

Pérez Otero, M. (2018). Vericuetos de la filosofía de Wittgenstein en torno al lenguaje y el seguimiento de reglas. Prensas de la Universidad de Zaragoza.

Wittgenstein, L. (1953). Philosophische Untersuchungen. Publicado originalmente en 1953. (Traducción: Investigaciones filosóficas, Crítica, 1988, edición bilingüe).

Wittgenstein, L. (1958). The blue and brown books. Basil Blackwell. (Traducción: Los cuadernos azul y marrón. Tecnos, 1968).

Wright, C. (1984). Kripke's account of the argument against private language. Journal of Philosophy, 81, 759-778.

Recibido el 2 de octubre de 2020; aceptado el 24 de noviembre de 2020. 